\title{
Engagement estratégico y encuentro conversacional en los medios sociales
}

\section{Strategic engagement and conversational encounter in social media}

\author{
Mariano Ure ${ }^{1}$ \\ Recibido: 03-10-2017 - Aceptado 20-12-2017 \\ DOI: https://doi.org/10.26441/RC17.1-2018-A10
}

\begin{abstract}
RESUMEN: El artículo aborda los supuestos de las metodologías para medir la conversación online. Estas registran lo que hacen los usuarios que acceden a perfiles de medios sociales institucionales, lo que permite observar la repercusión de las publicaciones. Sin embargo, no dan cuenta de la respuesta de las instituciones como resultado de la escucha a los usuarios, ni de las posibilidades de co-creación. Así, se confunde conversación con mera interacción digital. La búsqueda del engagement se ejecuta como una acción estratégica de conquista de usuarios, que termina por detener los avances hacia una cultura de la colaboración. El objetivo es ofrecer un aporte teórico que permita delinear los supuestos pragmáticos y éticos tanto de la interacción digital como del encuentro conversacional. Con ello, se cuestiona el paradigma conversacionalista, que identifica el intercambio lingüístico online como grado máximo de evolución de una comunicación social que aspira a lograr comprensión, cercanía y colaboración.
\end{abstract}

Palabras clave: medios sociales; engagement; conversación; interactividad; ética

ABSTRACT: The article approaches approach to the assumptions of methodologies for measuring online conversation. These methodologies record what users do when access to social media institutional profiles, which allows observing the impact of the publications. However, they do not take into account neither the response of institutions as a result of listening to users nor the possibilities of co-creation. Thereby, conversation is confused with mere digital interaction. Seeking for engagement is executed as a strategic action of conquest of users, which ends up stopping the advances towards a culture of collaboration. The objective is to offer a theoretical contribution that allows defining the pragmatic and ethical assumptions of both the digital interaction and the conversational encounter. With this, the conversationalist paradigm is criticized, which identifies the online linguistic exchange as the maximum degree of evolution of a social communication that pretends to achieve understanding, closeness and collaboration.

Keywords: social media; engagement; conversation; interactivity; ethics

1 Mariano Ure es Doctor en Disciplinas Filosóficas por la Universidad de Pisa (Italia), Magíster en Relaciones Internacionales Unión Europea - América Latina por la Universidad de Bolonia (Italia) y Licenciado en Filosofía por la Universidad del Norte Santo Tomás de Aquino (Argentina). Es profesor de la Licenciatura en Comunicación Periodística y Director del Programa de Investigación en Medios, Espacio Público y Culturas Digitales, en la Universidad Católica de Argentina. mariano_ure@uca.edu.ar, https://orcid.org/0000-0002-7222-7250 


\section{Introducción ${ }^{2}$}

El desarrollo y la rápida apropiación de las tecnologías de la comunicación digital durante la última década del siglo XX dieron lugar a una nueva utopía que dejaba atrás la desconfianza en la razón que había caracterizado el mundo occidental de posguerra. La "sociedad de la información", o sus más evolucionados conceptos de "sociedad del conocimiento" o "sociedad red", se postulaba como una tierra prometida que permitiría la solidaridad y la cooperación entre los seres humanos. Con gran optimismo acerca del carácter emancipatorio de estas tecnologías, distintos artistas, académicos y empresarios de Silicon Valley configuraron la Californian Ideology. Esta profesa una ideología digital en la que todos seríamos capaces de expresarnos libremente y, además, de desarrollar todo nuestro aletargado potencial creativo (Barbrook y Cameron, 1996).

Esta mirada tecnófila tiene como antecedente los estudios de Wiener (1949), McLuhan (1964) y Negroponte (1995), quienes sostenían que las tecnologías, y luego las computadoras, mejoraban sustancialmente la vida y potenciaban extraordinariamente las capacidades humanas. $\mathrm{Y}$ ya en el siglo XXI, el surgimiento de la Web 2.0 permitió una nueva concepción cultural sobre las dinámicas de intercambio de información. Ahora, los usuarios pueden organizarse en comunidades, resol- ver problemas de manera colaborativa y hacer circular contenidos compartiéndolos con otros usuarios (Jenkins, 2008). En efecto, tal utopía puede resumirse como la expectativa de avances cualitativos en el campo de las relaciones interhumanas a partir de incrementos sobre todo cuantitativos en el intercambio de información. El horizonte ideal es: el acceso, el intercambio, la producción y el compartir información y conocimientos. Esto permitiría la superación de límites e incapacidades, así como la supresión de inequidades y la facilitación de la comprensión recíproca.

En contrapartida, una postura más bien "apocalíptica", para usar los conocidos términos de Eco, comenzó a mostrar las contradicciones entre los ideales emancipatorios de la utopía de la interacción digital y las formas de manipulación y control emergentes. Detrás del imperativo del compartir, diseñado estratégicamente por las empresas que ofrecen servicios de comunicación interactiva, se esconde el interés de obtener distintos datos personales de los usuarios para ofrecérselos a los anunciantes. A pesar de que no se abone dinero para la suscripción, el servicio no es gratuito, sino que se paga a través de la autorización para el acceso a este tipo de datos. Esto hace que el producto sean los usuarios mismos (Magnani, 2014), para las empresas que lanzan sus campañas de marketing online. Al mismo tiempo,

2 Una primera versión de este artículo fue presentada y discutida como ponencia en la Conferencia IAMCR 2017. 
el uso intensivo de medios sociales configura una sociedad de la vigilancia, en la que instituciones públicas o privadas, e incluso miembros de la propia red de contactos (familiares, amigos, colegas, etc.), controlan nuestros gustos y actividades (Silverman, 2015). En efecto, la conexión humana en las plataformas es, en realidad, una "conectividad automatizada", ya que lo que hacemos se codifica en conceptos algorítmicos que luego se aplican para conducir nuestras futuras experiencias en ellas (Van Dijck, 2016). Esto se denomina trackeo, en el que el historial de nuestra navegación y nuestras acciones digitales quedan registradas como una huella, que luego los algoritmos inteligentes emplean para catalogar nuestra identidad. Estas prácticas son vistas como acciones que reducen la autonomía de los usuarios tanto por académicos como activistas, que ejecutan investigaciones e iniciativas educativas para advertir a los usuarios sobre la forma en la que los dispositivos y los servicios online los monitorean, como por ejemplo el proyecto Chupadatos (https://chupadados. codingrights.org/).

El artículo que desarrollamos se inserta en esta discusión acerca de los supuestos pragmáticos y éticos de las formas de conexión humana en el entorno digital, en particular en los medios sociales. Se trata de un abordaje crítico que se focaliza en las metodologías para medir la conversación online, las cuales registran lo que hacen los usuarios que acceden a perfiles de medios sociales institucionales, sin dar cuenta de la respuesta de las instituciones como resul- tado de la escucha a los usuarios, ni de las posibilidades de co-creación con ellos. Por lo tanto, estas metodologías parecen confundir la conversación con la mera interacción digital, donde la búsqueda del engagement se convierte en una acción estratégica de conquista de usuarios, que termina por detener los avances hacia una cultura amplia de la colaboración.

En el campo de la comunicación en redes sociales, el engagement es entendido como una práctica interactiva de un seguidor con una cuenta. La traducción literal, del inglés, significa "compromiso", y supone un grado de implicación emocional del usuario en reacción a los contenidos publicados en las redes sociales. En este sentido, el engagement puede definirse como una experiencia de usuario con tecnologías basadas en la web que lleva a los usuarios interactuar, crear y compartir contenido en sus redes (McCay-Peet y Quan-Haase, 2016). Allí, el usuario deja de ser un lector para convertirse en un actor del proceso de la comunicación, haciendo comentarios o compartiendo publicaciones (Harden y Heyman, 2009; Chandler y Munday, 2016). En este sentido, el engagement digital demuestra cierto interés del usuario por lo que otro está diciendo, así como también que está dispuesto a dedicar tiempo y energías a responder a otros usuarios o a decir algo sobre ellos (Evans, 2010; Goodman, 2012). Un usuario implicado ya no es indiferente frente al asunto que reúne a los usuarios, sino que reconoce que allí hay algo valioso a lo cual prestar atención, para luego 
decir algo también y, eventualmente, tomar alguna decisión o emprender una acción.

Para nuestro análisis crítico delineamos, en primer lugar, los hitos de la emergencia del paradigma conversacionalista en la comunicación digital, sus principales características y promesas, en contraposición al modelo informacionalista de la sociedad red. Luego, recorremos distintas fórmulas de medición del engagement, con sus respectivas métricas. Esto permitirá considerar si la búsqueda del engagement es una acción estratégica de viralización de contenidos que tiene como centro a la institución, en lugar del usuario. A partir de allí, se distinguen dos tipos de engagement, uno centrado en los intereses de quien publica, y otro que intenta la construcción de ámbitos de colaboración. Finalmente, ofrecemos un aporte teórico para la distinción entre dos prácticas, similares en términos de interactividad y opuestas en términos de reconocimiento humano: el engagement estratégico y el encuentro conversacional. Con ello, discutiremos si el conversacionalismo, concebido como intercambio lingüístico online, puede asumirse como el grado máximo de evolución de una comunicación social que aspira a lograr comprensión, cercanía y colaboración entre los interlocutores.

\section{Desarrollo}

\subsection{Emergencia del "conversaciona- lismo"}

Junto con el desarrollo de posibilidades técnicas de interacción en la web social, se fue configurando un nuevo paradigma y horizonte para la comunicación, que se puede denominar "conversacionalismo". El manifiesto Cluetrain (Levine, Locke, Searls y Weinberger, 1999) y la obra We Media (Bowman y Willis, 2003) mostraron que la comunicación involucra a muchos, sobre todo en calidad de productores de mensajes y de sentido. La actividad de los usuarios se vio aún más facilitada con el surgimiento de los medios sociales en la primera década del siglo XXI (Facebook 2004, Twitter 2006 e Instagram 2010). Allí los usuarios cuentan con la posibilidad de hacer publicaciones que expresen sus intereses, experiencias, ideas y opiniones. En la actualidad, la penetración de estos servicios es significativa, el $37 \%$ de la población mundial los utiliza activamente (Statista, 2017) y está conectado en promedio más de 2 horas diarias (Global Web Index, 2017). Por eso, distintas instituciones públicas, organizaciones privadas y medios de comunicación están ya lanzados a la esfera pública digital. El propósito general es establecer una relación más cercana y horizontal con su público, allí donde el público está.

La concepción conversacionalista se fue afianzando por impulso del periodismo y del marketing digital. El periodismo se presenta como una práctica conversacional que dejó atrás los discursos monológicos pronunciados como lecciones (Kunelius, 2001; King, 2008; Marchionni, 2013). Tradicionalmente, el periodista tenía un acceso privilegiado a la información, con lo cual se colocaba en cierto nivel de superioridad 
frente a su público. El desafío consiste, en cambio, en ofrecer espacios de participación en el que se entrecrucen la actividad periodística con las contribuciones informativas de los ciudadanos (Gillmor, 2004). Un paso hacia ese objetivo fue la posibilidad de que los usuarios hagan comentarios a las noticias y la publicación de la dirección de correo electrónico del periodista, así como también la creación de perfiles en medios sociales, que por otra parte constituyen una importante fuente de tráfico hacia los sitios de noticias (Anderson y Caumont, 2014). Sin embargo, la crítica habitual a estas iniciativas sigue siendo que los periodistas no consideran los comentarios y difícilmente responden al intento de los usuarios de entrar en contacto con ellos.

Al igual que para el periodismo, la actividad de las empresas en los medios sociales se volvió imprescindible para poder mejorar su imagen y lograr la fidelidad de sus clientes. El marketing digital se ocupa de ensayar y planificar estrategias de comunicación interactiva que favorezcan el sentimiento positivo del consumidor hacia la marca. Investigaciones en este campo sugieren que el engagement conversacional con la comunidad online de una marca se traduce en una mayor lealtad hacia ella, y que esto incide en el complejo proceso de decisión de compra (Dessart, Veloutsou y Morgan-Thomas, 2015). En los medios sociales, el engagement de los usuarios se entiende como participación, que puede ser o bien pasiva, a través del consumo de los contenidos, o bien activa, con la contribución o crea- ción de contenidos vinculados con las marcas (Taylor y Kent, 2014; Ashley y Tuten, 2015; Schivinski, Christodoulides y Dabrowski, 2016). El objetivo de gestión del marketing digital consiste en hallar las formas más eficaces para lograr el engagement de los usuarios objetivo, y así lograr en ellos los efectos deseados. En términos generales, las publicaciones informativas de las marcas provocan menos engagement que aquellos que se refieren a la personalidad de cada marca, con recursos humorísticos o emocionales (Lee, Hosanagar y Nair, 2015). Esta misma lógica proveniente del marketing digital, que busca involucrar a los usuarios para incrementar la difusión de las publicaciones y el sentimiento positivo, es utilizada además tanto por agencias de gobierno (Bhattacharya, Srinivasan y Polgreen, 2017) como por organizaciones sin fines de lucro (Syrdal y Briggs, 2016).

El paradigma conversacionalista se postula sobre esas bases como superador del "informacionalismo" propuesto por Castells (2009). Para el sociólogo catalán, la sociedad se configura en red y está integrada por nodos que procesan flujos. Estos flujos son corrientes de información que circulan por los canales que conectan a los nodos. Sin embargo, aun cuando la conversación se apoye necesariamente sobre unidades informativas que circulan entre los nodos-interlocutores, la conversación supone una diferencia cualitativa sustancial: en ella se establece reciprocidad e intercambio de roles entre hablante y oyente (Sacks, Schegloff y Jefferson, 1974; Stati, 1982), y permite la comprensión mutua 
(Gadamer, 1997). Gracias a la conversación, los interlocutores son capaces de escucharse y de compartir recursos para algún proyecto común.

\subsection{Las metodologías de medición del} desempeño en medios sociales

El ideal de la conversación como nueva forma de relación de proximidad con el público objetivo tiene como condición el engagement de los usuarios con las publicaciones de la institución o de la empresa. Si bien existen diferentes fórmulas para calcular el engagement, de acuerdo con el criterio de las empresas que ofrecen servicios de medición, algunos principios y métricas son comunes. En efecto, es insuficiente con tener tráfico, una gran cantidad de seguidores o un buen alcance (reach), sino que lo crucial es que los usuarios que acceden a los contenidos hagan algo con ellos en términos de interacción online (Raso, 2016). El engagement es, así, una medida del éxito de las publicaciones. Se lo considera como un efecto de aceptación y valoración de los contenidos por parte de los usuarios; una implicación emocional que se manifiesta explícitamente a través de los likes, comentarios y shares.

Núñez (2017) utiliza dos fórmulas para medir el engagement en determinado período: a) total de interacciones de los usuarios (likes, comentarios y shares) sobre la cantidad de personas que vieron la publicación x $100, \mathrm{y}$ b) total de interacciones sobre total de seguidores actuales x 100. Origami Logic (2016) calcula el índice de engagement considerando el número total de interacciones dividido por: seguidores (representa la audiencia que está potencialmente expuesta de manera directa a los contenidos), alcance (representa el número de individuos que estuvieron expuestos al contenido) o impresiones (representa el número total de vistas del contenido).

Otra manera de calcular el engagement es dividiendo el total de interacciones de los usuarios en un período de tiempo determinado por el número de publicaciones realizadas en ese mismo período y, luego, dividido por el número de seguidores, y multiplicado por cien (Human Level Communications). A continuación, mostramos las fórmulas para Facebook, Twitter e Instagram:

Gráfico 1: Fórmulas del engagement rate para Facebook, Twitter e Instagram

\section{f Engagement Rate facebook}

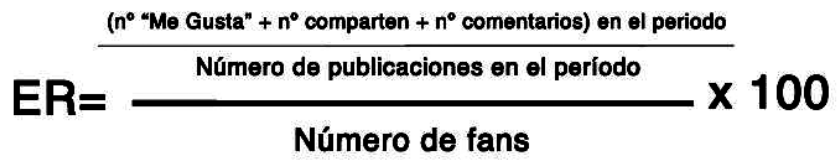




\title{
(y) Engagement Rate Twitter
}

\author{
( $n^{\circ}$ menciones $+n^{\circ}$ retuits) en el periodo \\ $\mathrm{ER}=\frac{\overline{\text { Número de tuits en el período }}}{\text { Número de seguidores }} \times 100$
}

ㅂ. Engagement Rate Instagram

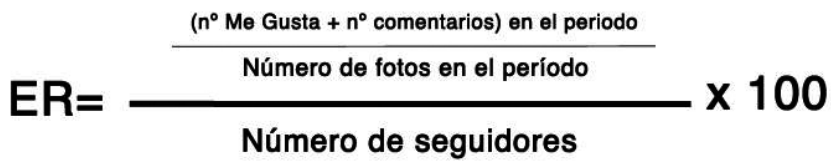

Fuente: Human Level Communications

Para Garst (2014), en cambio, el índice de engagement de Facebook surge de la cantidad de personas que dieron like, compartieron, comentaron o dieron click en los contenidos sobre la cantidad de personas que vieron las publicaciones. Estas métricas varían según la red social. En Twitter se consideran los likes, las menciones, las respuestas y los RT, mientras que en Instagram los likes y los comentarios. También para Fernández (2017) una buena medida del engagement tiene en cuenta el alcance y la interacción de los usuarios, pero excluye la cantidad de seguidores (fans, followers). El dato clave para conocer la efectividad de las acciones en medios sociales consiste en saber qué porcentaje de usuarios que están expuestos a ver un contenido publicado por una marca hicieron algo con ello.

Las interacciones de los usuarios con las publicaciones poseen diferentes ca- racterísticas. La primera es la del consumo del contenido (click). Si bien se trata de una acción que muestra interés por parte del usuario, no permite conocer su implicación emocional una vez consumido el contenido. Esto se sabe, en cambio, con las interacciones de aplauso, conversación y amplificación (Kaushik, 2011). El aplauso son las acciones que indican acuerdo, interés o empatía con la publicación (likes). La conversación incluye las respuestas directas o indirectas al contenido (comentarios, respuestas). La amplificación son las acciones por las que se comparte una publicación a una audiencia más extensa (shares, RT). El aplauso y la conversación representan interacciones que generan un engagement no viral y sirven para la retención de los usuarios e incrementar el ranking de las publicaciones en los algoritmos de las plataformas (Origami Logic, 2016). La 
amplificación permite aumentar el alcance de los contenidos, en este caso no a través campañas patrocinadas, que suelen tener menor eficacia, sino de la recomendación social, es decir, de los usuarios a sus propios contactos.

\section{Argumentación}

\subsection{Cultura de la colaboración y accio- nes estratégicas en las interacciones comunicativas}

Como muestran las distintas fórmulas, el índice del engagement funciona como una suerte de rating de interactividad. Los medios tradicionales como la prensa escrita y la audiovisual poseen sus propios índices para estar al tanto de su presencia en el mercado. Los datos sobre la circulación de medios impresos, el share de la audiencia de las emisoras de radio y el rating de los programas de televisión, dan cuenta de la aceptación del público a su propuesta comunicativa. En la web social, la circulación (alcance, impresiones) o el rating (clicks) no resultan datos suficientes, y son complementados con métricas sobre las reacciones de los usuarios que están expuestos a las publicaciones, de acuerdo con las posibilidades de interacción que ofrece cada una de las plataformas.

El mayor reto que enfrentan los profesionales que gestionan medios sociales es que crezca su comunidad de seguidores, y que estos se impliquen emocionalmente con la marca. Esto es, incrementar la presencia de la marca, mejorar su reputación y reforzar la lealtad de sus clientes. El asunto relevante para nuestra crítica consiste en preguntarse cómo valorar las acciones orientadas a incrementar el engagement y cuáles son sus posibles consecuencias. Habermas (1987) nos ayuda a interpretar la tendencia a medir el éxito del desempeño en los medios sociales a través del engagement, con la distinción entre acción estratégica y acción comunicativa. La primera está diseñada por el emisor para que el destinatario del discurso actúe de manera tal que se consigan los objetivos planificados previamente. La segunda es la acción que deriva de un intercambio discursivo que incluye a los interlocutores implicados, y que busca un bien común para todos ellos.

Siguiendo esta clasificación, el engagement en los medios sociales es un objetivo estratégico que busca cierta reacción de los usuarios: que los contenidos publicados por la marca (o la institución pública o sin fines de lucro) sean aceptados, apreciados y, sobre todo, compartidos (interacción de amplificación). En efecto, la estrategia de conquista de nuevos usuarios parece sostenerse en prácticas que se definen como conversacionales pero que, en realidad, se reducen a interacciones en reacción a la propuesta inicial de la marca, en las que no se realiza un intercambio de roles entre hablante y oyente. Esto trae como consecuencia una desaceleración hacia la promesa de la nueva cultura digital: la colaboración. Este horizonte supone la puesta en común de recursos (ideas, experiencias, conocimientos) para la co-creación. El simple compartir, que en las metodologías de cálculo del enga- 
gement se considera con la métrica de los shares y que constituye la reacción fundamental para la viralización de los contenidos, supone la reproducción de los contenidos, pero no la participación en la co-creación de bienes comunes.

Mientras la búsqueda del engagement configure una estrategia, cabe preguntarse también cómo es considerado el usuario implicado en esta conversación. Este es un punto crucial, ya que de acuerdo con la concepción del engagement que describimos anteriormente, el usuario queda reducido a promotor de contenidos en su propia red de relaciones, sin que se le ofrezca la oportunidad de participar activamente, con sus propios recursos, en la creación (tanto en la etapa de diseño como de realización) de algún bien común para la comunidad. Así, en lugar de ser reconocido en su subjetividad, es utilizado como herramienta para la amplificación de contenidos que responden al interés estratégico del emisor, por lo general político (instalación de agenda pública) o económico (incremento de rentabilidad).

\subsection{Engagement "de" y engagement "con"}

El cuanto compromiso e involucramiento, el engagement es un rasgo del diálogo. Sin embargo, tal como es entendido en el paradigma conversacionalista, es decir como acción estratégica de conquista de usuarios, genera una distancia que obstaculiza el encuentro interhumano. En efecto, no toda interacción en los medios sociales es dialógica. La potencialidad dialógica de las herramientas interactivas no es aún verdadero diálogo, el cual sirve a mitigar las relaciones de poder, a reforzar la dignidad y la autoestima, e involucra a los participantes en la toma de decisiones (Taylor y Kent, 2014). De hecho, el contacto facilitado por herramientas como Facebook, Twitter e Instagram, sigue siendo más bien de tipo unidireccional, de una organización hacia sus amigos o seguidores, en lugar de constituir un ámbito de encuentro conversacional. Así lo demuestran distintas investigaciones sobre el uso de plataformas sociales en los medios de comunicación y en las instituciones de gobierno (Cogo, 2012; Arata, 2014; Ure, 2016).

La distinción entre las dimensiones de lo interactivo y lo interhumano queda a la luz, además, en la medida en que tanto el engaño y la manipulación, así como también la solidaridad y la colaboración online, se llevan a cabo sobre prácticas interactivas. Lo que separa a unas y otras no es la medida de la eficacia o éxito en la reacción de los usuarios, calculada a través de distintas fórmulas y métricas, sino la actitud ética de quien publica los contenidos y la propuesta relacional que hace a sus destinatarios. El engagement supuesto en las metodologías de medición analizadas responde aún a un modelo institución-céntrico, a pesar de las interacciones digitales que se realizan y se miden.

Para ser más precisos sobre este tema, parece oportuno introducir una distinción conceptual entre dos tipos de engagement. Uno, justamente el calculado por los profesionales que gestionan 
medios sociales para instituciones y empresas, se puede denominar como engagement "de", mientras que el otro, de carácter ético, como engagement "con". El engagement "de" los usuarios responde al interés estratégico de que los propios contenidos sean aceptados y viralizados. Como desarrollamos anteriormente, allí se tiene en cuenta lo que hacen los usuarios con lo que dicen perfiles institucionales. Estas reacciones operan como el segundo acto de habla en una conversación (Ure y Parselis, 2013). Sin embargo, para que exista una conversación, entendida como intercambio, es aún necesario un tercer acto de habla, que consiste en la respuesta del primer interlocutor a la respuesta de su destinatario. En el tercer acto de habla se verifica que ambos interlocutores cumplieron los roles de hablante y oyente.

El engagement "con" también se lleva a cabo a través de interacciones. Sin embargo, allí el énfasis está puesto en lo que hacen las organizaciones con lo que hacen los usuarios. Esto implica una suerte de descentramiento en la que no se impone el interés de uno sobre el otro. Con esta actitud, el modelo institución-céntrico se convierte en un modelo usuario-céntrico. Este tipo de $e n$ gagement supone la actitud receptiva y atenta de escuchar lo que los usuarios tienen para decir, de manera que pueda establecerse un intercambio colaborativo sobre el asunto que los reúne (gobierno, servicios, información, etc.). Allí los usuarios son reconocidos como portadores de conocimientos, experiencias y opiniones relevantes. La rela- ción propuesta transforma a los usuarios de meros difusores a compañeros en objetivos comunes.

En este sentido, el engagement dialógico o encuentro conversacional "con" los usuarios representa un proceso bidireccional de dar y recibir que permite tomar decisiones que beneficien a todas las partes involucradas, no solamente a la organización (Taylor y Kent, 2014). El verdadero diálogo, como explica Buber (2006), requiere reconocimiento recíproco, simetría relacional, respeto y atención por lo que el otro tiene para decir. La respuesta a la interpelación del otro es entendida como responsabilidad ética frente a él, y donación de sí para atender a sus necesidades. Una actitud dialógica proyecta rostros sobre los actuales y potenciales consumidores de los propios actos de habla (publicaciones en los medios sociales), validándolos como compañeros con los que la organización tiene que colaborar para generar bienes comunes.

\subsection{El encuentro conversacional como comunicación dialógica}

La distinción conceptual entre los dos tipos de engagement permite abordar con más profundidad la pregunta acerca de si se puede asumir la conversación como el grado máximo de evolución de la comunicación a nivel social. Para ello es necesario, nuevamente, ensayar una clasificación terminológica que otorgue claridad a las dimensiones que intervienen cuando los usuarios se comunican a través de medios sociales. Esta tarea teórica es compleja y 
arriesgada, ya que las nomenclaturas resultan ineludiblemente arbitrarias, por lo tanto, queda siempre abierta la posibilidad de que sean otras. De todos modos, el valor reside en la capacidad de los términos, junto con su definición, para identificar prácticas cualitativamente diferentes, las cuales constituyen dimensiones clave en los procesos de comunicación.

Wolton (2010) aclara con agudeza que informar no es comunicar: la información existe por sí misma, mientras que la comunicación lo hace en la existencia del otro y en el reconocimiento mutuo. En este sentido, su concepción de la comunicación supera la explicación informacionalista de las redes desarrollada por Castells. Sin embargo, aún queda por abordar si la conversación, también ella superadora de la información, es comunicación. La respuesta a la problemática depende de qué se entiende concretamente por conversación, y de las relaciones entre los tipos de prácticas que conectan a los usuarios (gráfico 2).

En primer lugar, corresponde desarrollar el elemento fundamental de toda comunicación: la información. Está integrada por datos; es una posesión que puede moldearse y ordenarse; se agrega o recorta; se archiva; se envía o reenvía. En este sentido, la información opera en una dimensión cognitiva que no involucra éticamente a los interlocutores. Con nueva información los usuarios tienen la posibilidad de adquirir nuevos conocimientos y de ampliar su propio horizonte. Si bien en el entorno digital la información es entrega- da de manera unidireccional, es sumamente importante. En efecto, sobre la base de la información se pueden generar tanto conversaciones como comunicaciones. Toda publicación (o acto de habla) en medios sociales posee una información decodificable, un contenido que puede ser comprendido. A partir de la instancia de acceso a la información los usuarios son capaces de dar respuesta desde sus propios perfiles en los medios sociales. Sus respuestas conforman, a la vez, información, que es buscada y analizada por profesionales que gestionan medios sociales gracias a herramientas de monitoreo que producen big data.

La conversación, por su parte, es una práctica que busca el engagement "de" los usuarios. Sin embargo, parece adecuado introducir un matiz que permite identificar dos formas de entender la conversación. La primera se acerca más bien a la dimensión de la información. Allí la conversación es entendida como interacción online entre usuarios, en la que unos simplemente hacen algo con lo publicado por otro (clicks, likes, shares), es decir, los usuarios aceptan y reproducen la información inicial a partir de cierta implicación emocional. Esta conversación posee un objetivo estratégico y es institución-céntrica. La segunda, en cambio, se realiza como intercambio de información; apunta a la coordinación de acciones y opera en una dimensión pragmática-lingüística. Esta conversación como intercambio de contenidos es también concebida como participación (Sorice, 2009). Aquí, los usuarios son activos y producen contenidos, que son 
escuchados por la organización y considerados para la co-creación. Sin embargo, a pesar de que se establece el intercambio de roles entre hablante y oyente y de que es, básicamente, usuario-céntrica, esta conversación también puede ser estratégica, si es que la escucha se reduce exclusivamente al monitoreo de lo que hacen los usuarios en los medios sociales y que las respuestas de las organizaciones apuntan a mantener y hacer crecer su propia reputación.

Gráfico 2: Características y relaciones de los conceptos de información, conversación y comunicación

\begin{tabular}{|l|l|l|l|}
\hline \multicolumn{1}{|c|}{ INFORMACIÓN } \\
$\begin{array}{l}\text { Datos moldeables } \\
\text { Codificación y } \\
\text { acceso } \\
\begin{array}{l}\text { Distribución } \\
\text { unidireccional } \\
\text { Dimensión } \\
\text { cognitiva }\end{array}\end{array}$ & $\begin{array}{l}|c| \\
\text { Participación del hablante y el oyente } \\
\text { Apunta a un engagement estratégico como } \\
\text { conquista "de" los usuarios } \\
\text { Dimensión emocional y pragmática- } \\
\text { lingüística }\end{array}$ & $\begin{array}{l}\text { COMUNICACIÓN } \\
\text { Encuentro } \\
\text { conversacional } \\
\text { Requiere un } \\
\text { engagement } \\
\text { dialógico "con" los } \\
\text { usuarios } \\
\text { Interacción } \\
\text { Recesariamente } \\
\text { bidireccional } \\
\text { engagement } \\
\text { interactivo } \\
\text { Los usuarios } \\
\text { aceptan y } \\
\text { reproducen } \\
\text { contenidos } \\
\text { Modelo institución- } \\
\text { céntrico }\end{array}$ & $\begin{array}{l}\text { Intercambio } \\
\text { Requiere un } \\
\text { engagement } \\
\text { conversacional } \\
\text { Los usuarios } \\
\text { producen } \\
\text { contenidos y son } \\
\text { escuchados } \\
\text { Modelo usuario- } \\
\text { céntrico }\end{array}$ \\
\hline
\end{tabular}

Fuente: elaboración propia

La conversación como intercambio es soporte de la comunicación en la medida en que coloca a los interlocutores frente a sí, interesados en aquello que se están diciendo. Allí el contacto online se convierte en encuentro. En otras palabras, la comunicación es diálogo en el que, junto con el intercambio pragmático-lingüístico y más allá de este, se crea un ámbito de habitación común. Según esta perspectiva, el diálogo es un lugar antropológico de encuentro intersubje- tivo, que modifica a quienes participan en él. Su requisito esencial es el reconocimiento recíproco en cuanto interlocutores válidos, con legitimidad para hablar y con capacidad para hacer contribuciones relevantes. Ello está atado a la actitud, asumida libremente, de descentrarse a sí mismo para involucrarse "con" el otro. En efecto, la comunicación opera en una dimensión ética-comunitaria: hace posible la convivencia y la colaboración en proyectos compartidos. Para las 
organizaciones, entrar en diálogo implica intervenir en las conversaciones digitales con la pretensión de contribuir con los usuarios en la solución de problemas comunes, tanto los que surgen en la relación de consumo que los une inicialmente como aquellos más generales de la sociedad que ambos integran. Un signo de un diálogo auténtico consiste en que la organización modifique alguna de sus normas, procesos o servicios, a raíz de una propuesta o input de usuarios externos.

\section{Conclusiones}

La tensión entre una postura utópica y otra apocalíptica acerca de la omnipresencia de los medios sociales en el estilo de vida contemporánea es, por lo general, una oposición entre extremos que no da cuenta de la complejidad de lo que ocurre en el nuevo territorio digital. Allí emergen, al mismo tiempo, instancias de colaboración y de control; de reconocimiento y de engaño; de escucha y de imposición; etc.

El paradigma conversacionalista tampoco parece reparar en la simultaneidad de estas prácticas. Recostado sobre todo en los beneficios de la conexión online entre usuarios, no advierte lo suficiente acerca de la propensión de ciertos actores a la mercantilización y a la tecnificación de la comunicación. Las metodologías de medición del desempeño en medios sociales permiten registrar la repercusión de las publicaciones, una suerte de rating de interactividad. Sin embargo, no dan cuenta de la respuesta, producto de la escucha a los usuarios, ni de la colabo- ración en la co-creación. Así, el cálculo del engagement resulta válido para conocer la eficacia en la gestión estratégica de los perfiles en medios sociales. Sin embargo, parece desmedido asumir el engagement "de" los usuarios como muestra de un paradigma comunicativo conversacionalista, en el que todos pueden hacer valer sus ideas, experiencias, conocimientos y opiniones.

En efecto, las posibilidades de interacción online con sus respectivas métricas no resultan suficientes para afirmar que el periodismo o los mercados son actualmente conversaciones. Tampoco las estadísticas acerca del constante crecimiento de la cantidad de usuarios y el tiempo que están conectados a las plataformas sociales. Si bien la conversación supone la interacción, no se identifica necesariamente con ella. Para que exista conversación se requiere el intercambio de roles entre el hablante y el oyente. En este sentido, aun si la conversación se consolidara en tanto intercambio más allá de la simple interacción informativa, esta no agotaría la comunicación. La comunicación requiere involucramiento "con" el otro; un descentramiento de sí que da lugar al encuentro, del que derivan la convivencia comunitaria y la colaboración.

El desafío para las instituciones públicas y las empresas que poseen una presencia activa en los medios sociales consiste en transformar el ideal de la viralización, centrado en sus propios intereses, en el ideal de la colaboración en asuntos comunes. Las organizaciones, en efecto, no están atadas por una suerte de naturaleza a prácticas interactivas 
estratégicas, sino que pueden sumergirse en la escucha de los usuarios. Una escucha que no se identifique con el simple monitoreo de las publicaciones y del análisis del sentimiento de los usuarios en los medios sociales, sino como apertura real y disponibilidad a dejarse modificar por lo que ellos dicen. Las or- ganizaciones pueden hacerlo consultando ideas, y solicitando información y conocimientos. En este sentido, pueden generar espacios que las conviertan en curadoras de ideas y generadoras de comunidad. El horizonte ético, en este caso, es la colaboración, la co-producción y el co-gobierno.

\section{Bibliografía}

Anderson, M. y Caumont, A. (24 de septiembre de 2014). How social media is reshaping news [Pew Research Center]. Recuperado de: http://www.pewresearch.org/facttank/2014/09/24/how-social-media-is-reshaping-news/

Arata, G. (2014). Enti local e social network. Il Mulino, 2, 323-328.

Ashley, Ch. y Tuten, T. (2015). Creative Strategies in Social Media Marketing: An Exploratory Study of Branded Social Content and Consumer Engagement. Psychology \& Marketing, 32 (1), 15-27. Recuperado de: http://tracytuten.com/wp-content/uploads/2016/03/Ashley_et_ al-2015-Psychology_Marketing.pdf

Barbrook, R. y Cameron, A. (1996). The Californian ideology. Science as Culture, 1, 44-72. Recuperado de: http://www.comune.torino.it/gioart/big/bigguest/riflessioni/californian_ engl.pdf

Bhattacharya, S., Srinivasan, P., y Polgreen, Ph. (2017). Social media engagement analysis of U.S. Federal health agencies on Facebook. BMC Medical Informatics and Decision Making, 17:49.

Bowman, S. y Willis, CH. (2003). We Media: How audiences are shaping the future of news and information. The Media Center at The American Press Institute. Recuperado de: http:// www.hypergene.net/wemedia/download/we_media.pdf

Buber, M. (2006). Yo y Tú. Buenos Aires: Lilmod.

Castells, M. (2009). Comunicación y poder. Madrid: Alianza.

Chandler, D. y Munday, R. (2016). A Dictionary of Social Media. Oxford: Oxford University Press.

Cogo, G. (2012). I social network nella PA. Santarcangelo di Romagna: Maggioli.

Dessart, L., Veloutsou, C. y Morgan-Thomas, A. (2015). Consumer engagement in online brand communities: a social media perspective. Journal of Product \& Brand Management, 24 (1), 28-42. Recuperado de: http://www.emeraldinsight.com/doi/pdfplus/10.1108/ JPBM-06-2014-0635

Evans, D. (2010). Social Media Marketing: the next generation of business engagement. Indianapolis: Wiley.

Fernández, A. (4 de enero de 2017). Cómo calcular el engagement en social media [SocialMedier]. Recuperado de: http://socialmedier.com/formula-engagement-calcularengagement -rate-formula/

Gadamer, H.G. (1997). Verdad y método. Salamanca: Sígueme. 
Garst, K. (10 de mayo de 2014). Social Media Measurement and the Only Metric That Matters [Huffingtonpost]. Recuperado de: http:/www.huffingtonpost.com/kim-garst/social-media-measurement-_b_4910702.html

Gillmor, D. (2004). We the Media: Grassroots Journalism by the People, for the People. O'Reilly Media. Global Web Index (2017). Quarterly report on the latest trends in social networking. Recuperado de: http://insight.globalwebindex.net/hubfs/Reports/Social-Q1-2017/GWISocial-Summary-Q1-2017.pdf?t=1498575132979

Goodman, G.F. (2012). Engagement Marketing: How Small Business Wins in a Socially Connected World. Nueva Jersey: Wiley.

Habermas, J. (1987). Teoría de la acción comunicativa. Taurus: Madrid.

Harden, L. y Heyman, B. (2009). Digital Engagement: Internet Marketing That Captures Customers and Builds Intense Brand Loyalty. Nueva York: American Management Association.

Human Level Communications. Engagement. En Diccionario de Marketing Online. Recuperado de: https://www.humanlevel.com/diccionario-marketing-online/engagement

Jenkins, H. (2008). Convergence Culture. La cultura de la convergencia de los medios de comunicación. Barcelona: Paidós.

Kaushik, A. (10 de octubre de 2011). Best Social Media Metrics: Conversation, Amplification, Applause, Economic Value [Occam's Razor]. Recuperado de: https://www.kaushik.net/avinash/best-social-media-metrics-conversation-amplification-applause-economic-value/

King, K. (2008). Journalism as a Conversation. Nieman Reports, 62 (4), 11-13. Recuperado de: http://niemanreports.org/articles/journalism-as-a-conversation-2/

Kunelius, R. (2001). Conversation: a metaphor and a method for better journalism? Journalism Studies, 2 (1), 31-54.

Lee, D., Hosanagar, K. y Nair, H. (2015). Advertising Content and Consumer Engagement on Social Media: Evidence from Facebook. Working Paper No. 3087, Graduate School of Business, Stanford. Recuperado de: https://www.gsb.stanford.edu/faculty-research/working-papers/advertising-content-consumer-engagement-social-media-evidence

Levine, R., Locke, Ch., Searls, D. y Weinberger, D. (1999). Clutrain manifesto. Recuperado de: http://tremendo.com/cluetrain/

Magnani, E. (2014). Tensión en la red: libertad y control en la era digital. Buenos Aires: Autoría.

Marchionni, D. M. (2013). Journalism as a Conversation: A Concept Explication. Communication Theory, 23 (2), 131-147.

McCay-Peet, L. y Quan-Haase, A. (2016). A Model of Social Media Engagement: User Profiles, Gratifications, and Experiences. En O'Brien, H. y Cairns, P. (Eds.). Why Engagement Matters: Cross-Disciplinary Perspectives of User Engagement in Digital Media (199-217). Cham, Nueva York: Springer.

McLuhan, M. (1964). Understanding media: the extensions of man. Nueva York: McGraw-Hill. Negroponte, N. (1995). Being digital. Nueva York: Knopf.

Núñez, V. (17 de enero de 2017). Fórmulas para calcular el engagement en redes sociales y el CTR de tus publicaciones [Vilma Núñez]. Recuperado de: https://vilmanunez.com/ formula-engagement-redes-sociales-ctr/ 
Origami Logic (2016). The Marketer’s Guide to Measuring Social Engagement.

Recuperado de: https://resources.origamilogic.com/ebooks/

the-marketers-guide-to-measuring-social-engagement.

Raso, A. (28 de marzo de 2016). How to Measure Engagement the Right Way [Content Marketing Institute]. Recuperado de: http://contentmarketinginstitute.com/2016/03/ measure-engagement-right/

Sacks, H., Schegloff, E. A., y Jefferson, J. (1974). A simplest systematics for the organization of turn- taking for conversation. Language, 4, 696-735.

Schivinski, B, Christodoulides, G. y Dabrowski, D. (2016). Measuring Consumers' Engagement with Brand-Related Social-Media Content. Journal of Advertising Research, 56 (1), 64-80.

Silverman, J. (2005). Terms of service: social media and the price of constant connection. Nueva York: HarperCollins.

Sorice, M. (2009). Sociologia dei mass media. Roma: Carocci.

Stati, S. (1982). Il dialogo: considerazioni di linguistica pragmática. Nápoles: Liguori.

Statista (2017). Global social network penetration rate. Recuperado de: https:/www.statista. com/statistics/269615/social-network-penetration-by-region/

Syrdal, HA. y Briggs, E. (2016). Developing a Positive Organizational Image: The Role of Engagement with Social Media Content. SMA Proceedings, 74-83. Recuperado de: http://c.ymcdn.com/sites/www.marketingadvances.org/resource/resmgr/Proceedings/ SMA2016_v11.pdf\#page $=74$

Taylor, M. y Kent, M.L. (2014). Dialogic Engagement: Clarifying Foundational Concepts. Journal of Public Relations Research, 26 (5), 384-398.

Ure, M. y Parselis, M. (2013). Argentine Media and Journalists Enhancing and Polluting of Communication on Twitter. International Journal of Communication, 7, 2013, Feature 17841800. Recuperado de: http://ijoc.org/index.php/ijoc/article/view/2178/970

Ure, M. (2016). La comunicación de la administración pública en las redes sociales: los casos de las ciudades de Buenos Aires y Bolonia. Palabra Clave, 1, 240-270. Recuperado de: http://palabraclave.unisabana.edu.co/index.php/palabraclave/article/view/5156/pdf

Van Dijck, J. (2016). La cultura de la conectividad: una historia crítica de las redes sociales. Buenos Aires: Siglo Veintiuno.

Wiener, N. (1949). Cybernetics: or, Control and communication in the animal and the machine. Nueva York: Wiley.

Wolton, D. (2010). Informar no es comunicar. Barcelona: Gedisa. 